Article

\title{
Factors Affecting the Performance of Recommender Systems in a Smart TV Environment
}

\author{
Iftikhar Alam, Shah Khusro *(D) and Mumtaz Khan (D) \\ Department of Computer Science, University of Peshawar, Peshawar 25120, Pakistan; \\ iftikharalam@uop.edu.pk (I.A.); mumtaz.ksu@uop.edu.pk (M.K.) \\ * Correspondence: khusro@uop.edu.pk; Tel.: +92-34-4344-5008
}

Received: 15 April 2019; Accepted: 21 May 2019; Published: 27 May 2019

\begin{abstract}
The recommender systems are deployed on the Web for reducing cognitive overload. It uses different parameters, such as profile information, feedbacks, history, etc., as input and recommends items to a user or group of users. Such parameters are easy to predict and calculate for a single user on a personalized device, such as a personal computer or smartphone. However, watching the Web contents on a smart TV is significantly different from other connected devices. For example, the smart TV is a multi-user, lean-back supported device, and normally enjoyed in groups. Moreover, the performance of a recommender system is questionable due to the dynamic interests of groups in front of a smart TV. This paper discussed in detail the existing recommender system approaches in the context of smart TV environment. Moreover, it highlights the issues and challenges in existing recommendations for smart TV viewer(s) and presents some research opportunities to cope with these issues. The paper further reports some overlooked factors that affect the recommendation process on a smart TV. A subjective study of viewers' watching behavior on a smart TV is also presented for validating these factors. Results show that apart from all technological advancement, the viewers are enjoying smart TV as a passive, lean-back device, and mostly used for watching live channels and videos on the big screen. Furthermore, in most households, smart TV is enjoyed in groups as a shared device which creates hurdles in personalized recommendations. This is because predicting the group members and satisfying each member is still an issue. The findings of this study suggest that for precise and relevant recommendations on smart TVs, the recommender systems need to adapt to the varying watching behavior of viewer(s).
\end{abstract}

Keywords: smart TV; recommendations; recommender systems; group recommendations

\section{Introduction}

The Smart television (TV) is a connected device that provides an extended functionality in delivering digital contents, such as live channels, movies, dramas, shows, and video on demand (VOD) services [1]. The smart TV comes with processing capabilities, third-party platforms, operating systems, and media players. The amalgamation of television with a processor, connectivity capabilities, and support for the Web 2.0 features has made this device attractive not only for viewers but also for researchers [1]. As compared to traditional TV systems, smart TV is a computing device [1] that can perform a variety of operations, including voice and gesture recognition. The life cycle of the smart TV is longer as compared to other smart devices, such as a smartphone, smartwatch, laptop, etc., $[1,2]$. This motivates enterprises and companies to develop technologically-advanced hardware and software for smart TVs. However, numerous issues are associated with smart TVs, which include security and privacy, complex user interfaces, interactivity issues, bloatware [3], the complex nature of browsing and searching, and personalized recommendation issues [1]. In this paper, we targeted the issues and factors affecting the recommendation process in the context of smart TV watching scenarios. 
The most common approaches for watching the contents on smart TV are browsing and searching for desired contents from robust and diverse data sources, such as stored videos, live channels, clips, etc. However, such rich and growing data sources make it difficult to search for the desired contents [1]. The reasons include the (a) widely used legacy remote controls, (b) lean-back nature of the smart TV, (c) specialized entertainment device, and (d) a device for all types of viewers. The details of these reasons are (a) apart from the availability of a variety of input devices and smart remote controls, the legacy remote is still widely used with smart TVs and limits frequent interactions; (b) in the lean-back, the contents are normally enjoyed in passive mode and preferably less interactively; (c) the smart TV is a specialized entertainment device, normally used for watching video, movies, live channels, and playing games; (d) the smart TV is for every type of viewer, including senior citizens, non-technical persons, and kids. Hence, searching and browsing are among the difficult activities on smart TV [4]. Although electronic program guides (EPGs) may help in searching the desired channel, due to a significant collection of channels and programs on EPG the searching and scrolling is a difficult task [5]. In addition to searching and browsing, the recommender system helps in reducing information overload by helping a user to select the best items from a significant collection of items [6-8].

A recommender system is a software tool that recommends suitable items to a user or group of users [9]. The recommender systems infer user interests by utilizing various sources of data, such as user profiles, clicks, and feedbacks (rating, and like/dislike) [8,10]. However, in a smart TV environment, such data are neither accurate nor simple to predict or calculate because the smart TV represents a set of users with diverse interests and taste. This distinct watching behavior and purpose make smart TV a unique device; however, the recommender systems consider the smart TV as an ordinarily connected device and recommend items based on activities performed by a single user or group of users. Such approaches are neither viable nor accurate to recommend items to the exact viewer(s) of smart TV. Hence, in the context of smart TV viewing scenarios, content filtering, channel recommendation, scheduling programs, and personalized viewership are challenging opportunities [11].

The recommender systems use numerous approaches for recommendations. Examples of such approaches include content-based filtering, collaborative filtering, and hybrid approaches [12]. The content-based filtering techniques rely on the user's profile information and the item's profile information [13]. However, smart TV is normally enjoyed in groups, and hence, the user' profile information cannot represent the whole group of viewers. Therefore, content-based filtering is not suitable for a smart TV watching scenario. The collaborative filtering approaches rely on user feedbacks, i.e., implicit feedbacks and explicit feedbacks. The implicit feedbacks are calculated from user activities, such as navigation, browsing, etc.; whereas explicit feedbacks are provided by the user in the form of likes/dislikes, rating, etc., [14]. The explicit feedbacks are rarely provided by a smart TV viewer. Therefore, specifically in the context of smart TV, we are left with only a few implicit feedbacks for recommending relevant items to the viewer(s) [15]. In hybrid filtering techniques, both approaches are combined to get better recommendations. However, it suffers from the inherent issues of both content and collaborative filtering approaches. Although some hybrid recommender systems, such as discussed in [16], combined several techniques for achieving accuracy; yet, most of the techniques demand active feedbacks from the user, which is not welcomed by smart TV viewers due to lean-back nature of a smart TV. Moreover, we cannot expect the same interactions from viewers as they normally have with computers and smartphones [17]. In addition to the interactive nature of smart TV, it is enjoyed as a lean-back device and passive as legacy TV systems [18].

The approaches for analyzing user activities, such as data mining, clickstream analysis, and in-depth identification of a viewer(s) may lead to serious security and privacy threats [18]. Although security and privacy issues for smart TV are in the infancy stage, they are the most important concerns for smart TV viewers [19]. Security and privacy are usually ignored by the buyer, seller, and even by the manufacturer [1]. Such concerns need a thorough investigation to make smart TV more user-friendly and intelligent. As discussed, a smart TV can provide rich types of entertainment on a single platform; however, smart TVs are widely used for streaming live channels and movies [20,21]. This distinct nature 
puts a question mark on the performance of the existing recommender systems because these systems are specially designed for personalized recommendations on personalized devices, such as smartphones and personal computers. The feedback that comes from computers and smartphones can be handled by the existing recommender systems; however, the feedback that comes from smart TV needs further investigation for recommending relevant items to the viewer or group of viewers.

In the smart TV watching scenario, group recommendations play a vital role. This is because, in most of the households, the smart TV is enjoyed in a group for watching movies, dramas, news, etc., [22]. Different approaches are used for group recommendations, such as aggregated predictions and aggregated model for preferences aggregation of individuals in a group [22-24]. However, the approaches for the identification of group members is based on predictions and estimation. Furthermore, due to the diverse interest of individuals; aggregated predictions and preferences aggregation are not feasible solutions and may lead to privacy issues. Therefore, the exact identity of group members and satisfying every group member is still challenging.

This paper is an attempt to identify some overlooked factors that affect the recommendation process and recommendation results on a smart TV. The factors are validated by the results of a subjective study conducted for this research. The first factor that affects the recommendation process is the group of viewers in front of a smart TV. The recommender system considers smart TV as a single viewer; however, there may be groups having diverse interests. The second factor that affects the recommendation process is the limited provision of feedbacks by the viewer. The reasons for such limited provision of feedbacks include the lean-back nature of smart TV, legacy remote controls, and the shared nature of smart TV. The third factor that affects the recommendation results is the different watching behavior of smart TV viewers. The smart TV is normally used for watching movies, video, and live channels. The viewer rarely uses smart TV for reading news, books, articles, etc. Therefore, navigating between video, movies, and channels provide limited feedbacks to the recommender system. The app-based and complex user interfaces are the fourth factor that affects the recommendation process. The smart TV comes with an operating system and applications (apps in short). Therefore, navigating between channels means navigating between apps, which create hurdles in calculating the viewer(s) interests. The fifth factor that affects the recommendation process is the shared nature of smart TV. The focus of the recommender system is the delivery of personalized recommendations; however, smart TV is not a single user device (See Section 3 for more details).

The contributions of this study are:

- To investigate the issues and challenges in existing group recommender systems for content recommendations on Smart TV.

- To identify the factors that affect the performance of a recommender system in the context of smart TV watching scenarios.

- A subjective study for validating the factors by analyzing the watching behavior of smart TV viewers.

Results of the subjective study show that in most of the households, smart TV is enjoyed in groups for watching live channels, movies, and videos. Moreover, the identification of viewers for the personalized recommendation may lead to privacy concerns, which is not welcomed by the smart TV viewers. The findings of this paper suggest that the recommender system should treat smart TV as a different connected device. This is because the other devices, such as computers and smartphones, are personalized devices, whereas smart TV is a shared device. Furthermore, user modeling plays an important role in recommender systems [25] and may enhance the recommendation results. This paper further suggests an enhanced user and group modeling technique for enhancing the personalization services in general and group recommendations in specific on a smart TV.

The rest of the paper is divided into 7 sections. Section 2 is the state-of-the-art Literature on the recommender systems in the context of smart TV. Section 3 presents some Potential Factors that affect the recommendation process for smart TV viewers. Section 4 presents the Methods and Material for analyzing and validating the factors identified in Section 3. Sections 5 and 6 are Results and 
Analysis, respectively. Section 7 is Discussion and Future Research Work. Section 8 concludes the paper. References are listed at the end.

\section{Literature Review}

During 1950, TV was considered the primary source to influence public opinion and still plays a significant role in molding and shaping people's perception [26]. The legacy TV system is the most popular entertainment device still widely used [27]. Compared with the combined daily consumption of smartphones, tablets, and PC; the time spent on watching TV is still high [28]. Today's TVs are not only full-duplex in nature, but also smart and come with built-in operating systems, third-party software, and sensors [1]. Moreover, modern television, called smart TV, is significantly different from the technological point of view as well as from an entertainment point of view. The smart TV comes in traditional shapes and sizes as well as in the form of set-top-boxes (STBs) that can be connected with a variety of displays, including legacy TV systems. Smart TV has revolutionized the entertainment industries by enabling streaming of Web contents. This amalgamation opens new avenues not only for entertainment industries but also for education, health, defense, business, commerce, etc. The operating system of smart TV enables the installation of different software and for managing many distinct features related to connectivity, communication, and interaction with smart TV [29]. Table 1 shows and compares a list of the features of smart TV and legacy TV systems.

Table 1. A comparison of smart TV features with the legacy TV system.

\begin{tabular}{|c|c|c|c|}
\hline & Features & TV & Smart-TV/TV-Box \\
\hline \multirow{7}{*}{ Hardware perspectives } & Infrared & $\checkmark$ & $\checkmark$ \\
\hline & USB & $X$ & $\checkmark$ \\
\hline & Wi-Fi & $x$ & $\checkmark$ \\
\hline & Storage & $x$ & $\checkmark$ \\
\hline & HDMI & $x$ & $\checkmark$ \\
\hline & RJ45 & $x$ & $\checkmark$ \\
\hline & VGA & $x$ & $\checkmark$ \\
\hline \multirow{10}{*}{ Software perspectives } & Lean-back support & $\checkmark$ & $\checkmark$ \\
\hline & Personalization & $x$ & $\checkmark$ \\
\hline & Operating System & $X$ & $\checkmark$ \\
\hline & Mobile & $x$ & $x$ \\
\hline & Interactive & $x$ & $\checkmark$ \\
\hline & IP address & $x$ & $\checkmark$ \\
\hline & History/Logs & $x$ & $\checkmark$ \\
\hline & Third-party applications & $x$ & $\checkmark$ \\
\hline & Browser & $x$ & $\checkmark$ \\
\hline & Sensors support & $x$ & $\checkmark$ \\
\hline
\end{tabular}

As connected TV, a smart TV can stream a variety of contents from the Web. Because of this, the terrestrial broadcast TV channels are now shifting their TV contents to the Web. The channel owners have their own web applications, which can provide streaming services to their users. Similarly, video-sharing websites are available, such as YouTube, Netflix, etc. The videos to such video sharing websites are uploaded by the channel owner or by the third parties, including individuals.

Such a rich set of multimedia contents makes it difficult to search for the relevant content. Moreover, searching is a user-driven activity that mostly relies on user queries. In addition to searching for 
relevant content on a smart TV, the online recommender systems are used to overcome the cognitive overload and recommend different items to a viewer [30]. However, the existing recommender systems are designed for the individual user who consumes content on a variety of personal devices, including computers and smartphones. Recommendations on terrestrial TV are usually based on regions. TV operators recommend items, goods, and services on a regional basis. As recommendation is on regional bases, viewers have to watch the running content, or they simply change the channel. To avoid such stereotype TV content, viewers have shifted to more advanced watching systems, i.e., smart TVs. The smart TV gives better clues to the recommender systems because of an Internet Protocol (IP) address, the log file, from which location, interests, etc., can easily be extracted. However, it suffers from numerous issues (see Section 3 for details). Figure 1 shows a common recommendation process on smart TVs.

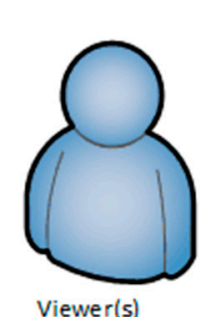

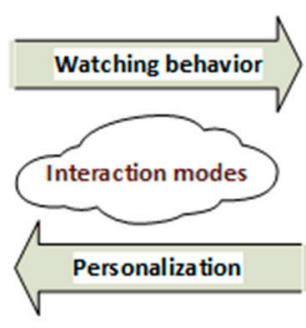

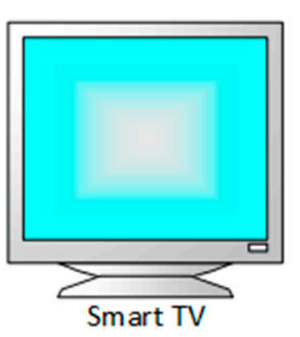

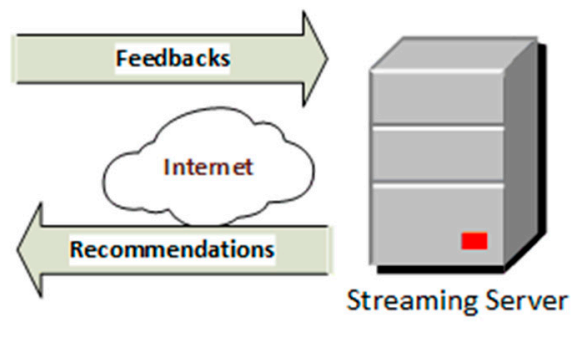

Figure 1. General scenario of recommendation process on a smart TV.

The following are some state-of-the-art systems and literature on recommender systems in the context of smart TV, connected TV, and Internet Protocol Television (IPTV).

In Hybrid Broadcast Broadband Television (HbbTV) project, they have designed several frameworks for identifying a viewer in front of a smart TV that include multi-user identification and multi-user recommendations $[17,18,31]$. HbbTV is heavily criticized in the literature for its security and privacy concerns as it is capable of capturing private data, such as picture and profile information [19]. Smart Parental Advisory [32] proposed a deep learning-based framework and usage control for implementing dynamic parental controls on a smart TV. The proposed work shows a camera with a TV for providing real-time parental control on smart TV content. In [33], a face recognition system for set-top-box based intelligent TV was proposed. They used a web camera for pictures, which is connected with a set-top-box, and a server is attached for face recognition. The exact identification of the viewer(s) on server-side is time-consuming due to the frequent switching of the viewer(s) in front of a smart TV. In [34], an enhanced recommender system was proposed by face detection and recognition system in front of a smart TV. They have used Face++ (https://www.faceplusplus.com/) and SkyBiomerty (https://skybiometry.com/) for face and emotion detection and recognition. Furthermore, they argued that detection of more than one person could form a group, and hence, the recommender system should recommend items to the group instead of individuals.

A TV program recommendation technique for a group of viewers was proposed in [35]. This work proposed a TV program recommendation system for multiple viewers (group) based on merging user profiles. Similarly, the study in [11] proposed the merging of multiple preferences to improve recommendation results. RecTime [36], proposed a real-time recommender for an online broadcasting system, which considers a user's preferences and time factors simultaneously. A personalized TV listings service for the digital TV [37] is proposed. The work describes the development of personalized television (PTV (http://www.ptv.e)) listing system which handles the information overload, by providing an Internet-based personalized listings service. Shinjee et al. [38], worked on the automatic and personalized recommendations of TV programs for smart TV viewers. They argued that due to the massive content available for watching on a TV, it is difficult to retrieve the desired program and hence, worked on the automation of recommending TV programs. An intelligent hybrid recommendation approach is proposed in [39], which uses hybrid collaborating filtering with a voice recognition system for controlling the smart TV. However, the work is limited to the recommendation of already crawled TV programs. Kwon and 
Hong [40], proposed a personalized program recommender system (PRS) for smart TV, which is based on a novel similarity method and collaborative filtering techniques.

Most smart TVs are equipped with automatic content recognition (ACR) system that automatically recognizes the content and recommend items to a viewer based on currently watching contents [41]. However, frequent switching of viewers in front of smart TV create difficulties for accurate recommendations. MovieLens (http://www.movielens.org) is an online web-based movie recommender system that invites viewer(s) to rate any movie in the list. In return, the system performs predictions and personalized recommendations. In the case of smart $\mathrm{TV}$, such types of web-applications are less effective because rating and tagging are among the difficult activities to perform on the legacy remote control of a smart TV. TV-Predicator [31], designed an application which allows personalized recommendations without disturbing the lean-back position in front of a TV. It uses the customers watching behavior and explicit feedbacks (ratings) on the server side to predict user preferences and recommend relevant items.

Different data-mining techniques are used to predict viewer preferences, such as, content-based filtering algorithms are used for related items, collaborative filtering techniques are used for ratings predictions, clustering techniques are used for increasing the performance, association rules mining approaches are used for analyzing item relations, vector space model is used for the identification of the viewer's watching patterns. However, data-mining techniques, clickstream analytics, and in-depth analysis of the viewer's data may lead to serious security and privacy issues [42]. We argue that personalization and recommendations on smart TV should not compromise the security and privacy of the viewers.

PolyLens [43], is a web-based movie recommender system that uses collaborative filtering techniques to recommend items to group users rather than individuals. PolyLens has used a group recommender extension to the MovieLens recommender system. PloyLens explores the design space of collaborative filtering recommenders for group viewers. A detailed log is maintained to measure how viewers formed a group(s). They also survey the group users and analyze their experiences of group recommendations. The issue with PolyLens is that it relies on collaborative filtering techniques. Collaborative filtering techniques suffer from data sparsity and gray sheep problems [44]. OntoTV [45] is developed for the management of different sources of TV contents. It uses semantic multimedia techniques by developing an ontology for different TV-related content. OntoTV is a television content management system, which retrieves content information from different sources and represents them using ontologies and knowledge engineering. A cloud-based program recommendation system (CPRS) [46] was proposed and implemented to improve channel recommendation systems by the formation of groups that have similar taste. The system is implemented using cloud computing, which uses the map-reduce algorithm. In CPRS, the first step is to cluster the user profiles and then grouped these profiles by the K-means clustering algorithm. These techniques required a good computation power to recommend an item from very large datasets.

J. Kim et al. [47] proposed a searching and recommendation method for TV programs based on contents and viewers ontologies. They designed the ontology model of TV programs to define the semantic structure of the content. Compared with keyword-based searching, a more precise searching was achieved on documentary programs. N. Chang et al. [28] proposed a TV program recommender framework, which integrates the Web 2.0 features into television sets and smart TVs (set-top-boxes). A personalized TV recommendation with mixture probabilistic matrix factorization [15] developed a two-stage framework for building a TV recommender system. First, the proposed framework automatically learns the number of watching groups, and then the mixture probabilistic matrix factorization (mPMF) model was proposed for learning the mixture preference of television programs. A TV program recommendation for multiple viewers based on users' profile merging techniques [29] was proposed. The work proposed a TV program recommendation for multiple viewers (group) based on merging user profiles. The profile merging is based on total distance minimization techniques that generate the results closed to most users' preferences.

Summarizing the literature; the content recommendation on a smart TV is based on a single user profile; however, in actual scenarios, the smart TV may be enjoyed in groups. This situation creates hurdles for existing recommendation algorithms. Although different approaches are used for group recommendations, such as aggregated predictions, preferences aggregation, etc., however, these approaches 
are not only difficult to calculate but also may lead to privacy issues. Furthermore, the identity of a group member is based on predictions and estimations and usually performed on the server-side, which may lead to serious privacy issues. The following section presents some potential factors that affect the personalization services, including the recommendations for smart TV viewer(s).

\section{Potential Factors}

In this section, we discuss the potential factors that may affect the recommendation process and recommendation results in the context of smart TV watching scenarios. These factors suggest that smart TV should be considered as a different device from other connected devices, such as computers and smartphones.

\subsection{Smart TV is a Shared Device}

In most households, smart TV is enjoyed in groups. The members of such groups may have different ages, gender, and taste. Therefore, recommending an item on smart TV may not be relevant to all group members. Moreover, the random switching of viewers in front of a smart TV makes it difficult to predict, maintain, and update multiple profiles on a smart TV. A smart-TV/STB registered with a single email address can be watched by the whole family members. Therefore, the recommendations based on login information may not be relevant. Moreover, there may be diverse preferences and interests, which makes it hard to predict the exact preferences of every individual in a group [15].

The group recommender system used two approaches for group recommendations, i.e., (i) aggregated predictions and (ii) the aggregated model for preferences merging of individuals [48]. These methods are based on predictions and estimations, and hence, the recommendations in such cases are not up to the mark. Moreover, the privacy concerns, and the varying interests of viewers, the preferences merging, and aggregate predictions are not practicable [49]. Figures 2 and 3 depict the aggregated predictions and aggregated model for preferences merging strategies.

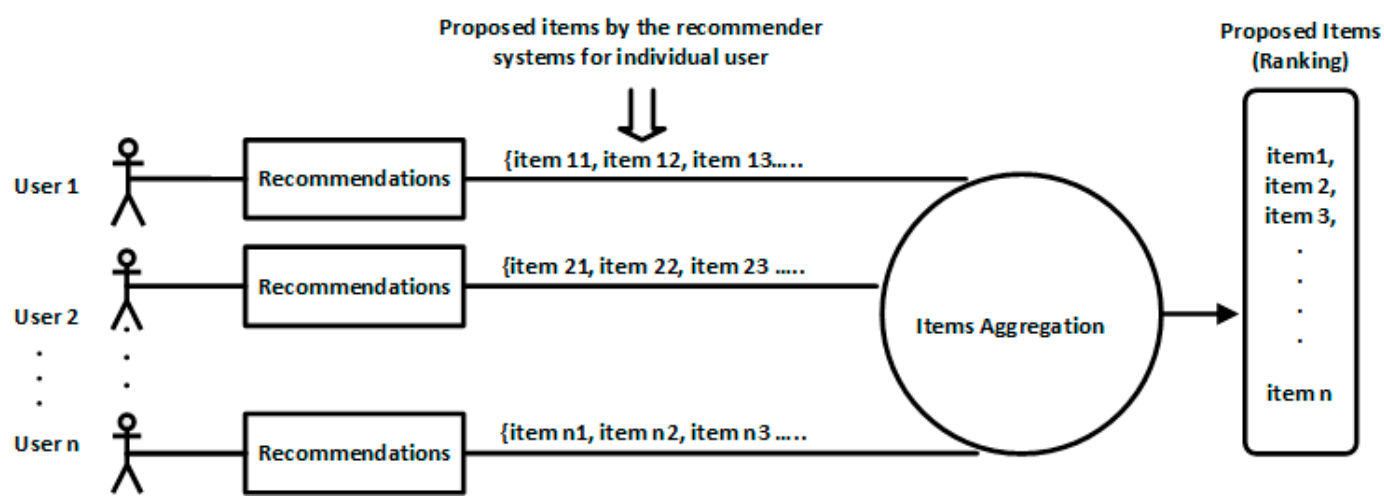

Figure 2. Aggregated prediction techniques in which items are aggregated for group recommendations.

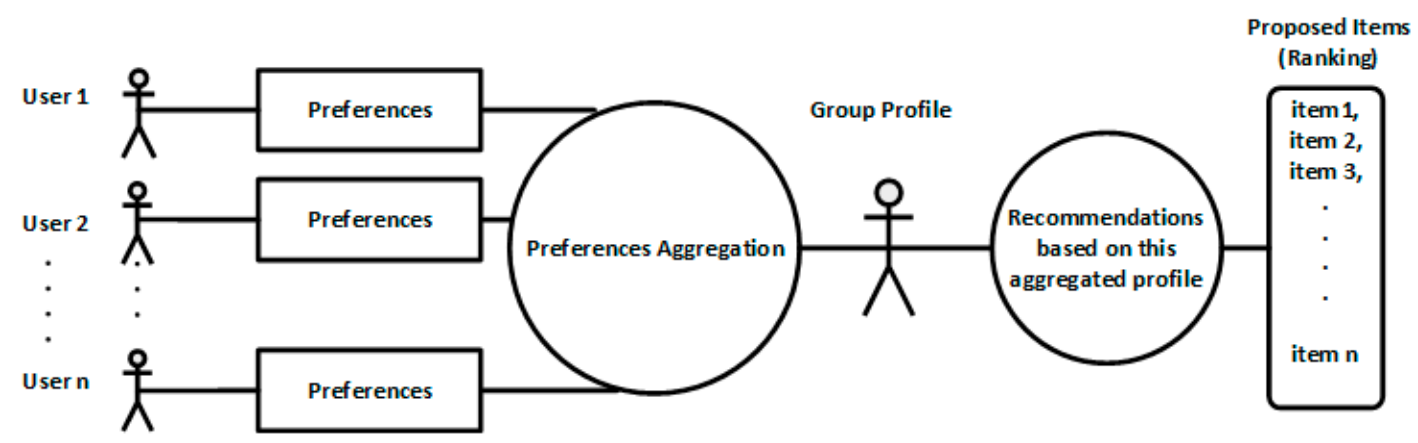

Figure 3. Aggregated model for preferences merging in which preferences of individuals are aggregated for group recommendations. 
In Figure 2, i.e., aggregated predictions, the recommendations to every individual are aggregated and ranked for recommendations to a group of users. In a smart TV watching scenario, merging the recommended items may lead to privacy issues. Hence, the recommendations based on aggregated predictions are not suitable for the smart TV watching scenario. In Figure 3, an aggregated model for preferences merging technique is depicted. In this technique, the preferences of users are aggregated for making a group profile. The recommender system then recommends items based on this group profile. The aggregated model for preferences merging is not viable due to the diverse interests of individuals. Moreover, calculating or predicting user preferences is not an easy task. Furthermore, identifying every individual of a group has not yet been achieved and is normally based on predictions. Therefore, in the smart TV watching environment, the recommendations based on preferences merging may not be easy or accurate. Therefore, the exact identity of viewer(s) and satisfying every individual of the group is still a challenging task.

\subsection{Limitations of Recommendation Approaches}

The recommender system uses different approaches for recommendations, such as collaborative filtering, content-based filtering, and hybrid approaches. The collaborative filtering approach take user feedbacks in the form of implicit, explicit, or combination of both [50]. The explicit feedbacks, such as rating and likes/dislikes, require explicit actions from a user; whereas implicit feedbacks are calculated by the recommender system from user activities [51]. The explicit feedbacks are difficult to provide in a smart TV environment [47]. Therefore, in a typical smart TV watching scenario, implicit feedbacks may be a better option than explicit feedbacks [18]. The second approach used by the recommender system is content-based filtering technique, which rely on the user's profile and item profile (description). In this technique, the recommender systems build a user profile; after which, the recommender system compares program attributes (item description) to a user profile and generates similarities between them [13]. Based on similarities, the suitable programs (items) are then recommended to a user. It uses watching history, likes, comments, and description with an object and recommends the best possible relevant item(s). When the description of an item matches with a user profile, history, etc., then an item is recommended to a user [52]. The issue that creates hurdles for content-based filtering algorithms is that smart TV is considered as a single profile (personalized device), such as a computer and smartphone. However, in most households, smart TV is enjoyed by an entire family or closed group [53]. In this case, the smart TV profile (single profile) may not be the true representative of the entire group/family [15]. In content-based filtering, the already consumed contents and profile play a key role in recommendations and hence, may fail to generate relevant and better results for the smart TV watching scenario. Furthermore, content-based filtering techniques rely on watching history; however, relying on watching history may not accurately recommend an item in the context of smart TV watching scenarios because of frequent switching of users. Furthermore, watching history cannot be related to every person in a group or family. Table 2 shows, some recommendation approaches, along with issues that may arise in recommending contents on a smart TV.

\subsection{Watching Behavior on Smart TV}

Smart TV is the most liked medium for watching the news, videos, games, music, etc., [54]. It can stream every available type of web content without time or location constraints. Despite the emergence of new gadgets to spend leisure time on the internet, TV watching remains the most popular activity around the globe [55]. However, the watching behavior on a smart TV is different from other devices, such as computers and smartphones. Despite the provision of lots of innovative features, smart TVs are mostly used for watching movies and video clips on the big screen. This watching behavior resembles with legacy TV systems, which is passive and less interactive. Hence, the expected feedbacks from a computer or smartphone user cannot be treated in the same manner with the feedbacks that comes from smart TV. We argue that instead of a pull mechanism, the recommender system should rely on a 
push mechanism for recommending item(s) for smart TV viewer(s). The pull mechanism needs active interactions with the device and hence, is not suitable for smart TVs.

Some social recommender systems (SRS) use social data for improving the recommendation results [56]. However, socializing on a smart TV is a rare activity. Despite the interactive nature of smart TVs, web 2.0 features, such as commenting, blogging, likes/dislikes, etc., are rarely used on a smart TV. Surfing web 2.0 features with a primary communication device (remote control) is a cumbersome job. As discussed, the main activity on a smart TV is to watch movies and videos on the big screen $[20,21]$.

Table 2. Recommendation techniques and issues in the context of smart TV watching scenarios.

\begin{tabular}{|c|c|c|c|}
\hline $\begin{array}{l}\text { Recommendation } \\
\text { Techniques }\end{array}$ & Approaches & Some Common Algorithms & Issues \\
\hline $\begin{array}{l}\text { Content-based } \\
\text { Filtering Techniques }\end{array}$ & $\begin{array}{l}\text { Item description, } \\
\text { user profile }\end{array}$ & $\begin{array}{l}\text { Cosine similarity, decision } \\
\text { tree, Bayesian network, } \\
\text { neural network, clustering } \\
\text { algorithms [12] }\end{array}$ & $\begin{array}{l}\text { Smart TV is not the true } \\
\text { representative of all viewers } \\
\text { behind smart TV [57] }\end{array}$ \\
\hline $\begin{array}{l}\text { Collaborative } \\
\text { filtering techniques }\end{array}$ & $\begin{array}{l}\text { Collective } \\
\text { preferences of the } \\
\text { crowd }\end{array}$ & $\begin{array}{l}\text { Cosine similarity, Pearson-r } \\
\text { correlation, Slope one, } \\
\text { Singular value } \\
\text { decomposition (SVD) [12] }\end{array}$ & $\begin{array}{l}\text { Unpredictable feedbacks in } \\
\text { case of smart TV watching } \\
\text { scenarios }\end{array}$ \\
\hline Hybrid Approaches & Combining Both & Any combination of above & Same inherited issues \\
\hline $\begin{array}{c}\text { Contextual } \\
\text { Recommendations }\end{array}$ & $\begin{array}{l}\text { Time. Place, location, } \\
\text { Events }\end{array}$ & $\begin{array}{c}\text { Contextual rules, Contextual } \\
\text { ontologies [12] }\end{array}$ & $\begin{array}{c}\text { Diverse interest of group } \\
\text { members }\end{array}$ \\
\hline
\end{tabular}

\subsection{TV Channel as an App}

The channels in smart TV are actually the apps that stream content from channel streaming servers or video sharing websites. Therefore, the contents are recommended within a specific app that is currently running. For example, YouTube recommends videos/clips within the YouTube app. Outside that app, the recommender system has nothing to do with other apps or contents. So, any channel (app) that has better recommendation algorithm will recommend items in a better way. We argue that recommendations should be app-independent.

\subsection{Smart TV User Interfaces}

Interaction modes play a vital role in the recommendation process because the interactions provide feedbacks to recommender systems. Smart TVs, including STBs, have full support for a variety of input devices including wireless keyboard and mouse. However, most of the viewers used the remote control as a primary communicating device with a smart TV. Hence, interacting by using a remote control is a difficult job, which in turn provide limited feedbacks to the recommender systems.

The explosion of recommended items may clutter the smart TV user interface. Cluttered user interface (UI) may make it difficult to search or open a channel [57]. What to recommend? and where to display the recommended item(s)? are the two major concerns for practitioners and UIs designers. The existing cluttered UIs of the smart TVs make it difficult for non-technical persons, especially senior citizens, to overview the recommended item(s) in detail and select the best one from the list. This phenomenon may contribute to cognitive overload [1]. We argue that the recommended item(s) should be displayed in such a way that enhances visibility and readability.

\subsection{Profile-Based Recommendations}

The main objective of most of the recommender system is personalized recommendations. For this purpose, all the activities of a user are tracked and logged. What a user has searched, watched, or watching is tracked by using profile information, cookies, login information, locations, IP address, a device address (MAC address), etc. Although these approaches work well for computer and 
smartphone users; however, it fails to accurately predict the viewers' preferences on smart TVs. A smart TV represents the entire group/family as a single profile; therefore, it is quite difficult to track multiple profiles behind this single device. Like the smartphone, smart TV can be registered by a viewer's email address for downloading the application from the app-stores. This email address (logging information) plays a vital role in the personalized recommendation. However, a smart-TV logged-in by a single person email address may be enjoyed by the entire group or family members, including a senior citizen, wife, husband, kids, etc. Furthermore, there may be many viewers, having diverse interests, ages, and preferences behind a single TV, which makes it difficult to predict the accurate preferences of a closed group or family [15].

\section{Validating the Factors}

For analyzing the watching behavior on smart TVs, we conducted a subjective study. The main purpose was to discover the factors that may affect the recommendation process and recommendation results on a smart TV. Although the watching behavior may be significantly different for every region of the world, we have tried to validate the factors that contribute to the recommendation process and ask the questions that possibly represent a larger population.

\subsection{Methods and Material}

A mixed mode survey, i.e., observation, interview, and questionnaire (attached as Annex-A in Supplementary Materials section), was used for data collection. A random sample of 300 viewers was selected from different age groups, including senior citizens, teenagers, housewives, professionals, educated, and literate. For making the survey unbiased, we surveyed every age group, including male and female. The grouping, demographics, percentage value, and standard deviation (SD) are represented in Table 3.

Table 3. Demographic information.

\begin{tabular}{|c|c|c|c|c|}
\hline Participants & Demographics & Number of Participants & Percentage & SD \\
\hline \multirow{2}{*}{ Gender } & Female & 90 & $30 \%$ & \multirow{2}{*}{84.85281} \\
\hline & Male & 210 & $70 \%$ & \\
\hline \multirow{4}{*}{ Age group } & 20 to 30 Years & 90 & $30 \%$ & \multirow{4}{*}{33.41656} \\
\hline & 31 to 40 Years & 95 & $31.66 \%$ & \\
\hline & 41 to 50 Years & 90 & $30 \%$ & \\
\hline & Others & 25 & $8.33 \%$ & \\
\hline \multirow{2}{*}{ Background } & Educated & 260 & $86.66 \%$ & \multirow{2}{*}{155.5635} \\
\hline & Literate & 40 & $13.33 \%$ & \\
\hline \multirow{4}{*}{$\begin{array}{l}\text { Smart TV Usage } \\
\text { Experience }\end{array}$} & 1 year & 110 & $36.66 \%$ & \multirow{4}{*}{37.19319} \\
\hline & 2 years & 95 & $31.66 \%$ & \\
\hline & 3 years & 70 & $23.33 \%$ & \\
\hline & Others & 25 & $8.33 \%$ & \\
\hline
\end{tabular}

There were 15 questions in the survey, in which four were related to personal information and excluded from results and analysis. The collected responses were filtered, in which the responses of 29 respondents were found invalid and hence, removed from the analysis. The rest of the responses of 271 respondents were used for analysis. The reliability of the questionnaire for 11 questions (items) was tested using the Statistical Package for Social Science (SPSS). Cronbach's Alpha value of 0.788 shows an acceptable and reliable score, as shown in Table 4. Moreover, the composite reliability (CR) with a score of 0.759 shows an acceptable range. The calculated value of the average variance extracted 
(AVE) was 0.28 , which is acceptable. The AVE was calculated after factor analysis (FA) using principal component analysis (PCA) in SPSS. The Root Mean Square Error (RMSE) value of 0.07, which is less than the recommended value of 0.08 , is also acceptable.

Table 4. Cronbach's Alpha score for the reliability of the questionnaire.

\begin{tabular}{ccc}
\hline Cronbach's Alpha & Cronbach's Alpha Based on Standardized Items & No of Items \\
\hline $\mathbf{0 . 7 8 8}$ & 0.831 & 11 \\
\hline
\end{tabular}

The descriptive statistics for each item are presented in Table 5 , which is generated by using factor analysis (FA) in SPSS. Moreover, the determinant value 0.013 from the correlation matrix is greater than 0.001 and shows a positive correlation between items. Furthermore, the Kaiser-Meyer-Olkin measure of sampling adequacy's value was 0.836 , which is greater than 0.7 and shows an appropriate factor analysis, as shown in Table 6.

Table 5. Descriptive statistics.

\begin{tabular}{cccc}
\hline & Mean & Std. Deviation & Analysis N \\
\hline Q5 & 1.4834 & 0.50065 & 271 \\
Q6 & 1.5203 & 0.50051 & 271 \\
Q7 & 1.4059 & 0.50681 & 271 \\
Q8 & 1.5461 & 0.58110 & 271 \\
Q9 & 2.1292 & 1.22396 & 271 \\
Q10 & 1.8339 & 1.31871 & 271 \\
Q11 & 1.3579 & 1.01156 & 271 \\
Q12 & 1.0480 & 0.21410 & 271 \\
Q13 & 1.8303 & 0.78926 & 271 \\
Q14 & 1.7085 & 0.77929 & 271 \\
Q15 & 1.4945 & 0.58923 & 271 \\
\hline
\end{tabular}

Table 6. Kaiser-Meyer-Olkin (KMO) and Bartlett's Test (df: Degree of Freedom, Sig: Significance).

\begin{tabular}{c|cc}
\hline Kaiser-Meyer-Olkin Measure of Sampling Adequacy & $\mathbf{0 . 8 3 6}$ \\
\hline \multirow{2}{*}{ Bartlett's Test of Sphericity } & Approx. Chi-Square & 1153.530 \\
\cline { 2 - 3 } & df. & 55 \\
\cline { 2 - 3 } & Sig. & 0.000 \\
\hline
\end{tabular}

\section{Results}

The following sub-sections present the results generated from the subjective study. The results are shown by using descriptive statistics, i.e., by using graph and charts. The main objective was to validate the factors that were discussed in Section 3. As discussed, the following results are based on 11 questions and responses of 271 respondents.

\subsection{Type of Smart TV}

From the collected responses, we found that most of the smart TV viewers (60\%) have set-top-boxes (STBs) at their homes, as shown in Figure 4a. One major reason is the low prices of STBs. Smart TVs with a built-in operating system are comparatively expensive. The collected data shows that viewers spent a good amount of daily time in front of a smart TV, as shown in Figure $4 \mathrm{~b}$. However, the time spent may vary due to different events, vacations, mood, etc. As shown in Figure $4 \mathrm{~b}$, most of the viewers spent a reasonable amount of daily time, i.e., 1 to $8 \mathrm{~h} /$ per day in front of a TV. This shows that apart from personalized gadgets, such as smartphones, the importance of TV watching cannot be ignored. 


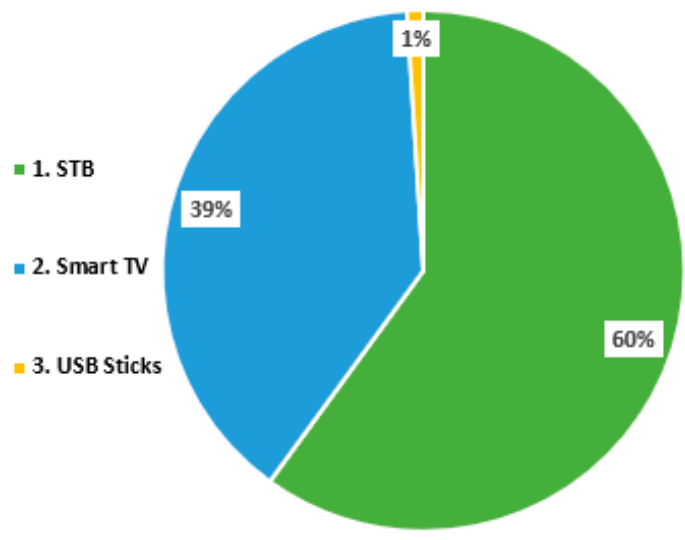

(a)

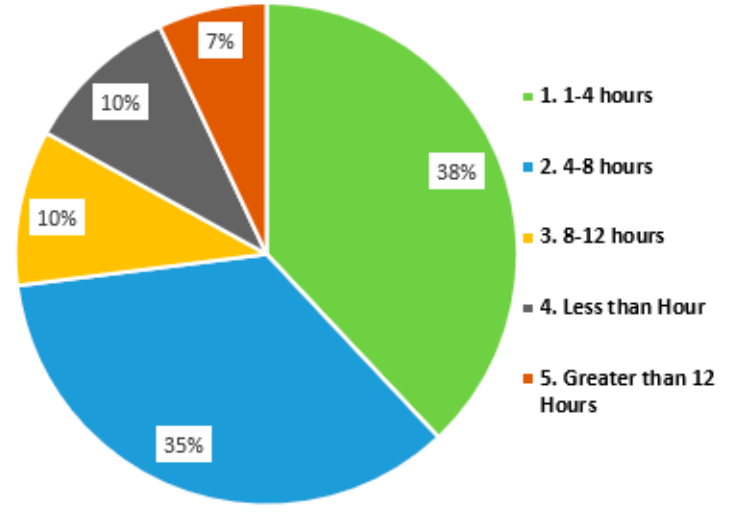

(b)

Figure 4. (a) Type of smart TVs; (b) Time spent in front of a smart TV.

\subsection{Watching Activities}

We found that watching videos, movies, clips, and game playing are among the major activities on smart TVs. Other activities, such as socializing, checking or sending emails, reading books/blogs, etc., are rarely used on smart TVs. This shows that being technologically advanced, smart TVs are treated as a legacy TV system. After applying the multiple correspondence analysis (MCA), we obtained Figure 5, which shows that streaming live channels and video watching are the most dominant activities on a smart TV. Moreover, smart TVs are enjoyed by either group or mixed (sometimes individual + sometimes group). It further shows that apart from full support for the Web 2.0 features; it is rarely used on smart TVs due to its shared nature and privacy issues. Analyzing the collected data, we found that viewers used smart TVs for watching moving pictures (videos, clips, etc.) on big High Definition (HD) screens.

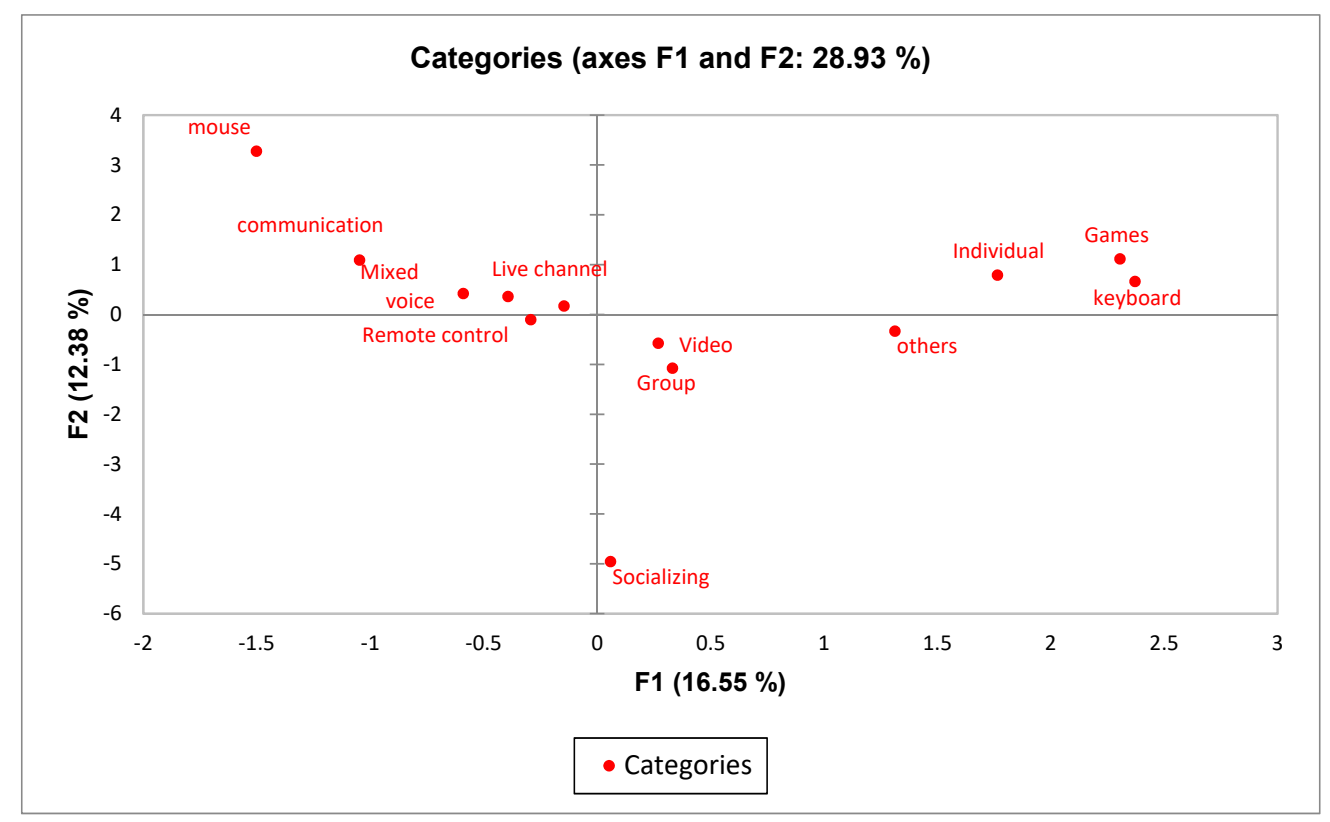

Figure 5. Multiple correspondence analysis (MCA) results for analyzing major activities on a smart TV.

\subsection{Privacy Concerns}

From the collected data, we found that privacy and security are big concerns for smart TV viewers. Not surprisingly, 95\% of viewers refused to allow a smart TV to keep track of personal information for personalized services, including recommendations. Figure 6 shows a high average rate for privacy concerns, which is 4.4 out of 5 . This shows that personalization services, including recommendations 
at the cost of security and privacy, are not welcomed by the viewers. Moreover, the leakages of TV viewership data may compromise the privacy of the whole family or closed groups.

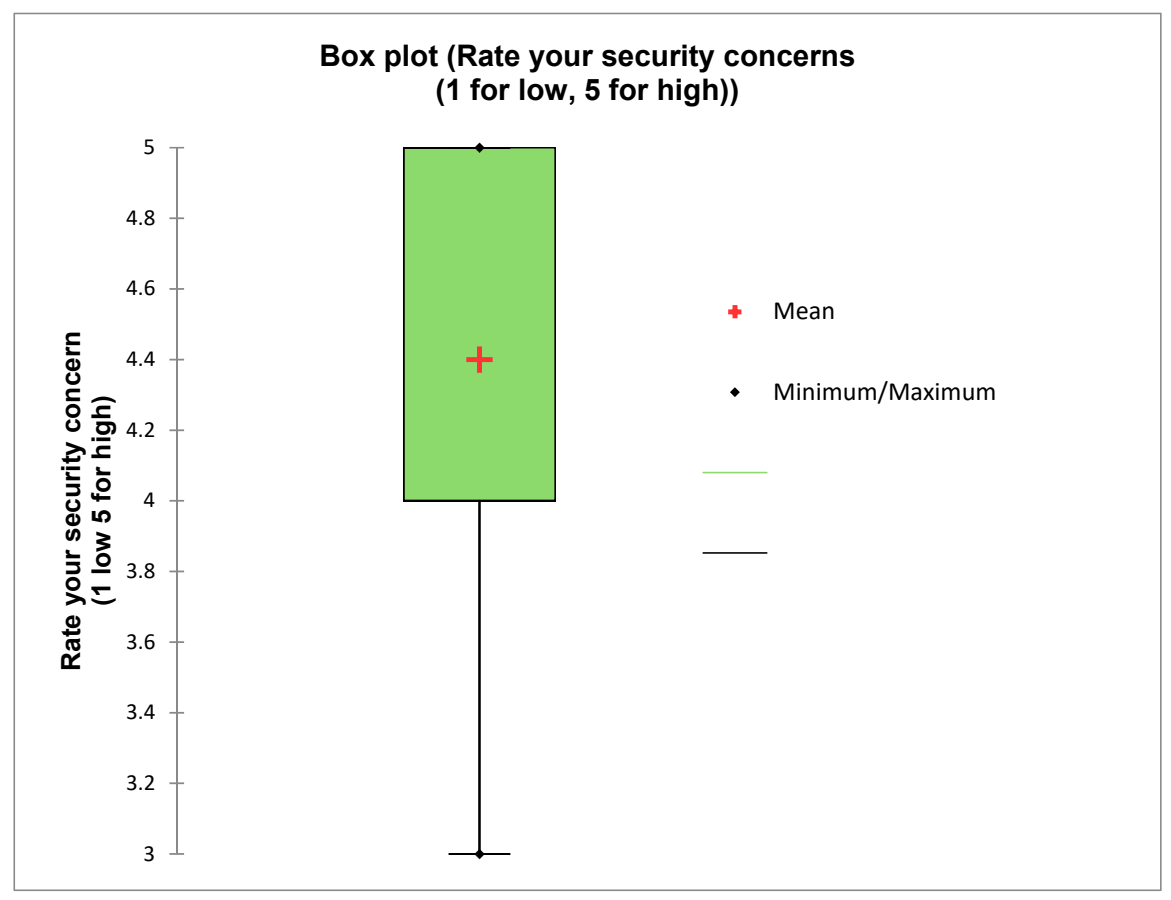

Figure 6. Box plot, showing high-security concerns by smart TV viewers.

\subsection{Primary Communication Device with Smart TVs}

The primary device for interactions with a smart TV is a remote control used by $86 \%$ of viewers, as shown in Figure 7a. This shows that writing blogs, commenting, likes/dislikes, and textual entry is a cumbersome task on this primary device, i.e., remote control. Moreover, we found that voice-based commands are used only by $5 \%$ of viewers, as shown in Figure $7 \mathrm{~b}$. One reason is that English was not the native language where we collected the data. For example, instead of using the Google Assistant on smart TVs, the press and clicks were preferred methods for retrieving the desired contents. Although smart remote controls, including smartphone-based remote controls, are available, these smart remote controls are either expensive, device dependent, or complex to use. That is why the legacy remote controls are still widely used. An easy to use a universal smart remote control for superseding the legacy remote controls is yet to come.

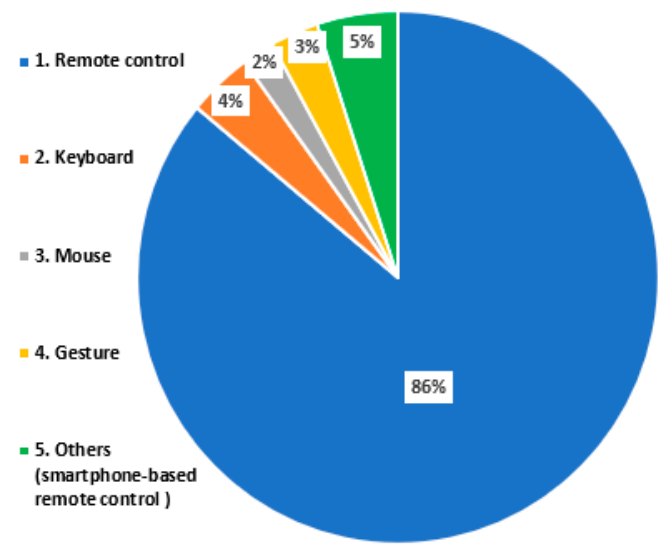

(a)

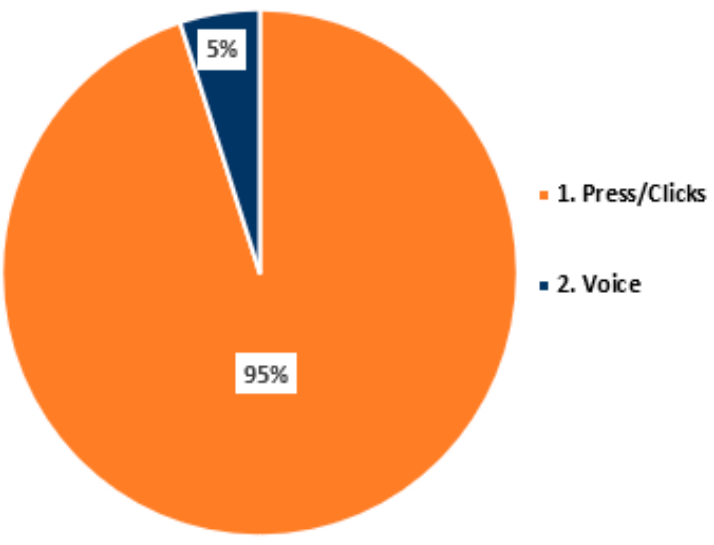

(b)

Figure 7. (a) Primary interaction device for smart TV; (b) Preferred method of interaction. 


\subsection{Device Registration Via Email}

The device registration plays a vital role in personalized recommendations. Asking about device registration was a bit of a technical question for viewers; however, we achieved our desired results. We found that Android-based smart TVs and Apple TV boxes are registered with a personal email by $35 \%$ of viewers shown in Figure 8a. Such registration is mandatory for downloading applications from app stores, such as Google-Play-store (https://play.google.com/store/apps), iTunes (https://itunes.apple.com/us/genre/ios/id36? $\mathrm{mt}=8$ ), etc. This registration information is considered as explicit feedbacks for recommender systems. However, the registration of a smart TV with a single email is not viable because this TV can be enjoyed by other people as well. Moreover, we found that $31 \%$ of viewers have downloaded applications from app-stores, as shown in Figure 8b. The apps, such as live channels and games, are the most downloaded categories on a smart TV. This shows that smart TV viewers are interested mostly in entertainment-related apps, such as live channels, videos, and games.

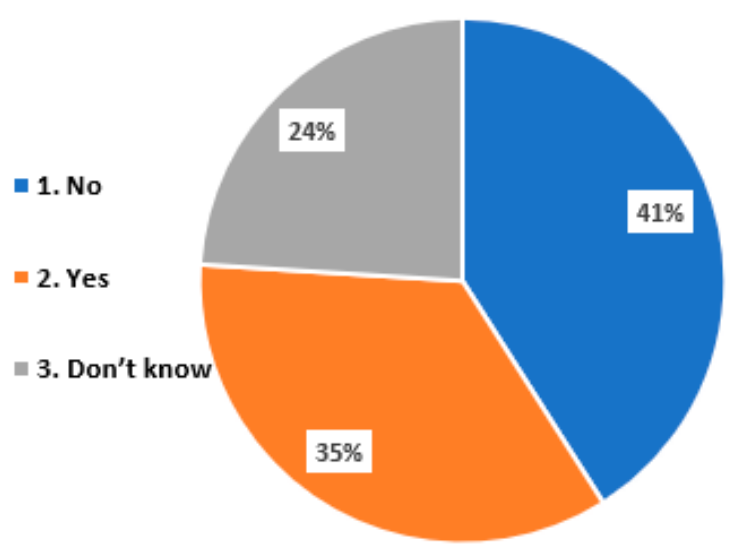

(a)

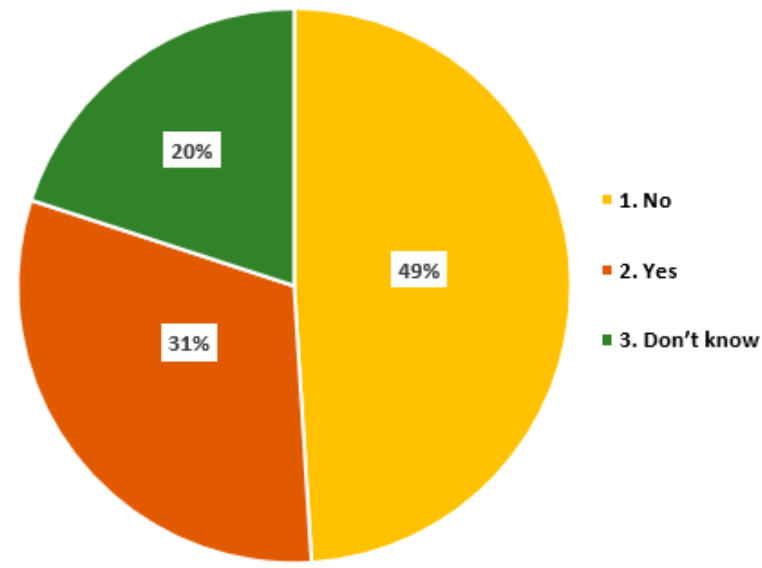

(b)

Figure 8. (a) Device Registration Via Email; (b) Downloading Apps from App-stores.

\section{Analysis}

The results of this study confirm that watching behavior on a smart TV is different from other connected devices, including computers and smartphones. Moreover, apart from the technological advancement in smart TV technologies, it is used as traditional TV. Furthermore, it is confirmed that smart TV is enjoyed in groups as a passive, lean-back device, in which the viewer(s) prefer less interactivity with a smart TV. These factors provide limited input to recommender systems. Thus, the recommendation results generated on smart TV may be irrelevant to different viewers in front of a smart TV. Rest of the findings are analyzed in the following sub-sections.

\subsection{Primary Activity on Smart TV}

A smart TV can stream almost every type of Web content, including live channels, movies, clips, web 2.0 features, etc. However, as discussed, the smart TV is enjoyed as traditional TV. Analyzing the collected data, we found that viewers used smart TVs for watching moving pictures, i.e., videos, clips, etc. Although the transformation of the legacy TV system to smart TV took a long time, people are enjoying smart TV as a lean-back device with lots of innovative features and options for watching their desired TV contents. This watching behavior provides very limited clues for a personalized service, including recommendations. As discussed, in most of the households, the smart TV is used as a shared device, i.e., a device for the whole family. However, the existing recommender systems are not intelligent enough to consider the whole family or closed group member for precise and relevant recommendations. Therefore, the recommended item(s) may not be relevant to all viewers in front of a 
smart TV. From the subjective study, it is confirmed that writing blogs, commenting, likes/dislikes, and other textual entry is a cumbersome task by using a remote control. Therefore, the recommender systems relying on user feedbacks for recommendations may not perform well for smart TV viewers.

\subsection{Passive Feedbacks}

The existing recommender systems take a user feedbacks, preferences, and watching history as input and recommend items to a user. The feedbacks may be explicit or implicit. The explicit feedbacks, such as commenting, likes/dislikes, login information, etc., are among difficult activities on smart TV (see Section 3 for more details). The implicit feedbacks are normally calculated from user activities, such as browsing, navigations, clicks, etc. In a smart TV watching scenario, the calculation or estimation of implicit feedbacks are not only difficult but also inaccurate results are highly probable. The reasons include the lean-back nature of smart TV. Moreover, behind a smart TV, we have multiple profiles that make it difficult to calculate and estimate the exact viewer profile. As discussed, television watching is a passive activity. The viewers prefer less interaction during content consumption on smart TV. The legacy remote controls and complex user interfaces further restrict the interaction with a smart TV. This type of passive feedbacks and watching behavior provides very limited input to recommender systems. Surfing the web contents on computers and smartphones are significantly different from a smart TV. However, the existing recommender rarely considers such factors during the recommendation process.

\subsection{Personalized Recommendations and Privacy Concerns}

The private data of a viewer may be captured for the delivery of personalized services, such as VOD services and recommendations. Results of the subjective study show that viewers are reluctant to allow a smart TV to capture their private data. Some recommender systems, such as used by $\mathrm{HbbTV}$ project of Hbb-Next, capture the private data of viewers, such as face detection and recognition for making precise recommendations. However, the HbbTV has been criticized in the literature for capturing a viewer's private data. This study suggests a tradeoff between recommendation results and privacy concerns. We argue that the recommender systems should seamlessly recommend items without any breaches of security and privacy.

\subsection{Primary Communication Device with Smart TV}

Analyzing the collected data, we found that apart from technological advancement, the primary communication device with a smart TV is a legacy remote control. Apart from full support, other communication devices, such as wireless keyboards, mouse, touch pads, etc., are rarely used with a smart TV. Although smartphone-based remote controls are also available; however, more accessible and innovative smartphone-based remote controls are still to come. Moreover, this study confirms that the key press is a major activity on the remote control for retrieving desired contents. Voice-based commands are used only by $5 \%$ of viewers. Some reasons for this low usage of voice-based commands are poor multi-lingual support, noisy environment, the pronunciation of words, etc. Although advanced technologies, such as Apple's Siri for Apple-based STBs, Google-Assistants for Android-based smart TVs, and smartphone-based universal remote controls are available; however, they are rarely used for watching smart TV contents.

\section{Discussion and Future Work}

The significant and growing TV-related multimedia content on the web makes it difficult to find relevant contents and hence, leads to content, information, and cognitive overload. For these reasons, recommender systems are used to help a viewer in selecting relevant contents. The recommender system considered different parameters for recommendations, such as user feedbacks, watching history, profile information, and user's preferences [16]. All these parameters are significantly different in the perspectives of smart TV watching scenarios. For example, user feedbacks are difficult due to the lean-back nature of smart TV. Similarly, smart TV is a multi-user device and hence, profile 
information, watching history, and user preferences are difficult to calculate. Therefore, the existing recommendation process needs a thorough investigation for relevant recommendations to the smart TV viewer(s). This paper presents some issues and challenges, along with some critical factors that affect the recommendation process on a smart TV. In the context of smart TV watching scenarios, the existing recommender systems have numerous issues and challenges, such as (1) the passive lean-back nature of a smart TV, (2) group/shared device, (3) connected devices that may leak a viewer's privacy, (4) remote-control as primary device for communication that restricts frequent interactions. Moreover, the nature of content delivery on a smart TV is based on a push mechanism that makes it difficult to provide better clues for recommender systems. The pull mechanism, in which the user is actively involved in the interaction, is not well suited for smart TV.

In the future, we intend to expand this work for semantically enriched contextual recommendations on smart TVs. We aim to design a recommender system for smart TV viewers that relies mostly on viewer's implicit feedbacks rather than explicit feedbacks. Furthermore, we aim to design a dedicated recommender system for smart TV viewers that have the least security and privacy issues. Most of the recent research work ignores the user-centric recommendations, which may play a major role in smart TV recommendations. A better study of smart TV watching behavior and viewer(s)/group modeling may further enhance the recommendation results. Social metadata should be used in such a way that further enhance the recommendations on a smart TV. Although some work [58] has already been done by integrating the social metadata with recommender systems for enhancing recommendation results for TV programs, more work is needed for incorporating social metadata with a recommender system for mitigating the issues of feedbacks on a smart TV. It is worth mentioning here that the social metadata of every family member is not necessary to be available every time. Therefore, social metadata should be integrated in such a way that it represents the whole group instead of individuals. The results of the subjective study suggest that better user or group modeling techniques may further enhance the recommendation results. Moreover, this study suggests the design of a more innovative and easy-to-use smart remote control for a smart TV that may have better control over smart TV's dynamic user interfaces.

\section{Conclusions}

This paper highlighted some overlooked issues that affect the performance of the recommender system in the context of smart TV environment. Some future research guidelines and potential factors were also presented for the improvement of existing recommender systems. The results of this study confirmed that for precise and relevant recommendations on a smart TV, the existing approaches are neither enough nor accurate to cope with the dynamic watching behavior of the viewer or group of viewers. The factors have been validated by a subjective study of smart TV viewers. Behind a smart $\mathrm{TV}$, we have multiple profiles that have diverse interests. The recommender system should understand these multiple profiles (groups) and the interests of individuals for relevant recommendations on a smart TV. We argue that better user modeling or group modeling may enhance the performance of the recommender system in a typical smart TV environment. Moreover, we argue that rich contextual information, such as vacations, the day of weeks (holidays, office timings), events (Christmas, Eid, Diwali, Ramadan, Sports events, World cups, etc.) should be integrated in such a way as to enhance the performance of existing recommendation approaches. We further argue that recommender systems should treat smart TV as a different device from other personalized devices, such as computers and smartphones.

Supplementary Materials: Annex-A: Questionnaire available online at http://www.mdpi.com/2227-7080/7/2/41/s1.

Author Contributions: Conceptualization, I.A. and S.K.; Data curation, M.K.; Formal analysis, I.A. and M.K.; Investigation, I.A.; Methodology, I.A. and S.K.; Project administration, S.K.; Software, I.A.; Supervision, S.K.; Writing - original draft, I.A. and M.K.; Writing - review \& editing, S.K.

Funding: This research received no external funding.

Conflicts of Interest: The authors declare no conflict of interest. 


\section{References}

1. Alam, I.; Khusro, S.; Naeem, M. A review of smart TV: Past, present, and future. In Proceedings of the International Conference on Open Source Systems and Technologies (ICOSST' 2017), Lahore, Pakistan, 18-20 December 2017; pp. 35-41.

2. Privitera, D.; Shahriar, H. Design and development of smart TV protector. In Proceedings of the National Cyber Summit (NCS'18), Huntsville, AL, USA, 5-7 June 2018; pp. 45-52.

3. Alam, I.; Khan, M.A.; Naeem, M.; Khusro, S. There is no such thing as free Lunch: An Investigation of Bloatware Effects on Smart Devices. J. Inf. Commun. Tech. Robot. Appl. 2017, 8, 20-30.

4. Jeong, J.-W.; Lee, D.-H. Inferring search intents from remote control movement patterns: A new content search method for smart TV. IEEE Trans. Consum. Electron. 2014, 60, 92-98. [CrossRef]

5. Yu, C.; Ding, H.; Cao, H.; Liu, Y.; Yang, C. Follow me: Personalized IPTV channel switching guide. In Proceedings of the 8th (2017)ACM on Multimedia Systems Conference, Taipei, Taiwan, 20-23 June 2017; pp. 147-157.

6. Gorgoglione, M.; Panniello, U.; Tuzhilin, A. Recommendation strategies in personalization applications. Inf. Manag. 2019. In Press. [CrossRef]

7. Roy, A.; Banerjee, S.; Bhatt, C.; Badr, Y.; Mallik, S. Hybrid Group Recommendation Using Modified Termite Colony Algorithm: A Context Towards Big Data. J. Inf. Knowl. Manag. 2018, 17, 1850019. [CrossRef]

8. Isinkaye, F.; Folajimi, Y.; Ojokoh, B. Recommendation systems: Principles, methods and evaluation. Egypt. Inform. J. 2015, 16, 261-273. [CrossRef]

9. Hamada, M.; Hassan, M. Artificial Neural Networks and Particle Swarm Optimization Algorithms for Preference Prediction in Multi-Criteria Recommender Systems. Informatics 2018, 5, 25. [CrossRef]

10. Reusens, M.; Lemahieu, W.; Baesens, B.; Sels, L. A note on explicit versus implicit information for job recommendation. Decis. Support Syst. 2017, 98, 26-35. [CrossRef]

11. Kim, M.S.; Kim, S. Factors influencing willingness to provide personal information for personalized recommendations. Comput. Hum. Behav. 2018, 88, 143-152. [CrossRef]

12. Véras, D.; Prota, T.; Bispo, A.; Prudêncio, R.; Ferraz, C. A literature review of recommender systems in the television domain. Expert Syst. Appl. 2015, 42, 9046-9076. [CrossRef]

13. Hsu, S.H.; Wen, M.H.; Lin, H.C.; Lee, C.C.; Lee, C.H. AIMED-A personalized TV recommendation system. In Proceedings of the European Conference on Interactive Television (EuroITV 2007), Amsterdam, The Netherlands, 24-25 May 2007; pp. 166-174.

14. Hanna, P. Considering Durations and Replays to Improve Music Recommender Systems. EAI Endorsed Trans. Artif. Intel. 2019, arXiv:1711.05237. [CrossRef]

15. Li, H.; Zhu, H.; Ge, Y.; Fu, Y.; Ge, Y. Personalized TV Recommendation with Mixture Probabilistic Matrix Factorization. In Proceedings of the International Conference on Data Mining (SIAM' 2015), Vancouver, BC, Canada, 30 April-2 May 2015; pp. 352-360.

16. Burke, R. Hybrid recommender systems: Survey and experiments. User Model. User-adapted Interact. 2002, 12, 331-370. [CrossRef]

17. Shin, J.; Park, Y.; Lee, D. Google TV or Apple TV?-The Reasons for Smart TV Failure and a User-Centered Strategy for the Success of Smart TV. Sustainability 2015, 7, 15955-15966. [CrossRef]

18. Van Deventer, M.; de Wit, J.; Guelbahar, M.; Cheng, B.; Marmol, F.G.; Köbel, C.; Köhnen, C.; Rozinaj, G.; Stockleben, B. Towards next generation Hybrid broadcast broadband, results from FP7 and HbbTV 2.0. In Proceedings of the IBC 2013, Amsterdam, Netherlands, 13-17 September 2013.

19. Michéle, B. Smart TV Security: Media Playback and Digital Video Broadcast; Springer: Cham, Switzerland, 2015.

20. Perakakis, E.; Ghinea, G. Smart Enough for the Web? A Responsive Web Design Approach to Enhancing the User Web Browsing Experience on Smart TVs. IEEE Trans. Hum. Mach. Syst. 2017, 47, 860-872. [CrossRef]

21. Soskic, N.; Vranic, N.; Lukic, N. Improving user experience with unique channel list on hybrid set-top boxes. In Proceedings of the IEEE International Workshop on Consumer Electronics (CEWS'15), Novi Sad, Serbia, 11 March 2015; pp. 46-47.

22. Felfernig, A.; Boratto, L.; Stettinger, M.; Tkalčič, M. Group Recommender Systems: An Introduction; Springer: Boston, MA, USA, 2018.

23. Akerkar, R. Recommendation Engines. In Artificial Intelligence for Business; Springer: Cham, Switzerland, 2019; pp. 41-52. 
24. Villavicencio, C.; Schiaffino, S.; Diaz-Pace, J.A.; Monteserin, A. Group recommender systems: A multi-agent solution. Knowl. Syst. 2019, 164, 436-458. [CrossRef]

25. Sánchez, P.; Bellogín, A. Building user profiles based on sequences for content and collaborative filtering. Inf. Process. Manag. 2019, 56, 192-211. [CrossRef]

26. WikiPedia. Televission. Available online: https://en.wikipedia.org/wiki/Television (accessed on 7 May 2019).

27. Yoong, S.; López, G.; Guerrero, L.A. Smart Device-Based Notifications: A Survey on User's Satisfaction of Traditional Notification Mechanisms. In Proceedings of the International Conference on Applied Human Factors and Ergonomics (2017), Los Angeles, CA, USA, 17-21 July 2017; pp. 104-114.

28. Mela, Y.D.; Carl, F. TV Viewing and Advertising Targeting. J. Market. Res. 2018, 55, 99-118. [CrossRef]

29. Michéle, B.; Karpow, A. Watch and be watched: Compromising all smart tv generations. In Proceedings of the 2014 IEEE 11th Consumer Communications and Networking Conference (CCNC), Las Vegas, NV, USA, 10-13 January 2014; pp. 351-356.

30. Ricci, F.; Rokach, L.; Shapira, B. Recommender systems: Introduction and challenges. In Recommender Systems Handbook; Springer: Boston, MA, USA, 2015; pp. 1-34.

31. Krauss, C.; George, L.; Arbanowski, S. TV Predictor: Personalized Program Recommendations to be displayed on SmartTVs. In Proceedings of the 2nd International Workshop on Big Data, Streams and Heterogeneous Source Mining: Algorithms, Systems, Programming Models and Applications (2013), Chicago, IL, USA, 11 August 2013; pp. 63-70.

32. Giorgi, G.; La Marra, A.; Martinelli, F.; Mori, P.; Saracino, A. Smart Parental Advisory: A Usage Control and Deep Learning-Based Framework for Dynamic Parental Control on Smart TV. In Proceedings of the International Workshop on Security and Trust Management (2017), Oslo, Norway, 14-15 September 2017; pp. 118-133.

33. Lee, W.O.; Kim, Y.G.; Hong, H.G.; Park, K.R. Face recognition system for set-top box-based intelligent TV. Sensors 2014, 14, 21726-21749. [CrossRef]

34. De Pessemier, T.; Verlee, D.; Martens, L. Enhancing recommender systems for TV by face recognition. In Proceedings of the 12th International Conference on Web Information Systems and Technologies (WEBIST 2016), Rome, Italy, 23-25 April 2016; pp. 243-250.

35. Yu, Z.; Zhou, X.; Hao, Y.; Gu, J. TV program recommendation for multiple viewers based on user profile merging. User Model. User-Adapted Interact. 2006, 16, 63-82. [CrossRef]

36. Park, Y.; Oh, J.; Yu, H. Rectime: Real-time recommender system for online broadcasting. Inf. Sci. 2017, 409, 1-16. [CrossRef]

37. Smyth, B.; Cotter, P. A personalised TV listings service for the digital TV age. Knowl. Syst. 2000, 13, 53-59. [CrossRef]

38. Pyo, S.; Kim, E.; Kim, M. Automatic and personalized recommendation of TV program contents using sequential pattern mining for smart TV user interaction. Multimed. Syst. 2013, 19, 527-542. [CrossRef]

39. Yang, Y.; Liu, C.; Li, C.; Hu, Y.; Niu, Y.; Li, L. The recommendation systems for smart tv. In Proceedings of the International conference on Computing, Communication and Networking Technologies (ICCCNT'14), Hefei, China, 11-13 July 2014; pp. 1-6.

40. Kwon, H.J.; Hong, K.S. Personalized smart TV program recommender based on collaborative filtering and a novel similarity method. IEEE Trans. Consum. Electron. 2011, 57, 1416-1423. [CrossRef]

41. Schelp, K.; Zheng, D. Linear Sales Automation and Data Insight As a Step Towards Programmatic TV. In Proceedings of the Embracing Connective Media (SMPTE'17), Sydney, Australia, 18-20 July 2017; pp. 1-12.

42. Deventer, O.V.; Wit, J.D.; Vanattenhoven, J.; Guelbahar, M. Group recommendation in an Hybrid Broadcast Broadband Television context. In Proceedings of the 20th Conference on User Modeling, Adaptation, and Personalization (UMAP 2012), Montreal, QC, Canada, 16-20 July 2012.

43. Connor, M.O.; Cosley, D.; Konstan, J.A.; Riedl, J. PolyLens: A Recommender System for Groups of Users. In Proceedings of the Seventh European Conference on Computer Supported Cooperative Work, Bonn, Germany, 16-20 September 2001.

44. Yang, Q. A novel recommendation system based on semantics and context awareness. Computing 2018, 100, 809-823. [CrossRef]

45. Redondo-Garcia, J.L.; Lozano-Tello, A. OntoTV: An ontology-based system for the management of information about television contents. Int. J. Semant. Comput. 2012, 6, 111-130. [CrossRef] 
46. Lai, C.-F.; Chang, J.-H.; Hu, C.-C.; Huang, Y.-M.; Chao, H.-C. CPRS: A cloud-based program recommendation system for digital TV platforms. Future Gener. Comput. Syst. 2011, 27, 823-835. [CrossRef]

47. Kim, J.-M.; Yang, H.-D.; Chung, H.-S. Ontology-based recommender system of TV programmes for personalisation service in smart TV. Int. J. Web Grid Serv. 2015, 11, 283-302. [CrossRef]

48. Masthoff, J. Group recommender systems: Combining individual models. In Recommender Systems Handbook; Springer: Boston, MA, USA, 2011; pp. 677-702.

49. Kompan, M.; Bielikova, M. Group recommendations: Survey and perspectives. Comput. Inf. 2014, 33, 446-476.

50. Pu, P.; Chen, L.; Hu, R. Evaluating recommender systems from the user's perspective: Survey of the state of the art. User Model. User-Adapted Interact. 2012, 22, 317-355. [CrossRef]

51. Jawaheer, G.; Szomszor, M.; Kostkova, P. Comparison of implicit and explicit feedback from an online music recommendation service. In Proceedings of the 1st international workshop on information heterogeneity and fusion in recommender systems, Barcelona, Spain, 26 September 2010; pp. 47-51.

52. Lu, J.; Wu, D.; Mao, M.; Wang, W.; Zhang, G. Recommender system application developments: A survey. Decis. Support Syst. 2015, 74, 12-32. [CrossRef]

53. Goren-Bar, D.; Glinansky, O. FIT-recommending TV programs to family members. Comput. Graph. 2004, 28, 149-156. [CrossRef]

54. Velusamy, S.; Gopal, L.; Bhatnagar, S.; Varadarajan, S. An efficient ad recommendation system for TV programs. Multimed. Syst. 2008, 14, 73-87. [CrossRef]

55. Sánchez, A. Digital Services in the 21st Century: A Strategic and Business Perspective; John Wiley \& Sons: Hoboken, NJ, USA, 2017.

56. Margaris, D.; Vassilakis, C. Exploiting Rating Abstention Intervals for Addressing Concept Drift in Social Network Recommender Systems. Informatics 2018, 5, 21. [CrossRef]

57. Gorla, J.; Lathia, N.; Robertson, S.; Wang, J. Probabilistic group recommendation via information matching. In Proceedings of the 22nd international conference on World Wide Web (2013), Rio de Janeiro, Brazil, 13-17 May 2013; pp. 495-504.

58. Shin, C.; Woo, W. Socially aware TV program recommender for multiple viewers. IEEE Trans. Consum. Electron. 2009, 55, 927-932. [CrossRef] 